\title{
EMDR in the Treatment of Medically Unexplained Symptoms: A Systematic Review
}

\author{
Yanda R. van Rood \\ Leiden University Medical Centre, The Netherlands \\ Carlijn de Roos \\ Psychotrauma Center for Children and Youth, The Netherlands
}

\begin{abstract}
This systematic review presents evidence for the effectiveness of eye movement desensitization and reprocessing (EMDR) in the treatment of a diverse range of medically unexplained symptoms (MUS). Theoretical underpinning, variations in interventions, methodological issues, and outcomes are discussed, and implications for future research and clinical practice are presented. Considering the limited number of reported case series and the lack of controlled studies, it might be concluded that EMDR for MUS is only in its infancy. The preliminary results suggest that EMDR might be an effective treatment for MUS and somatoform disorders, particularly when they are related to trauma. To date, the results for phantom limb pain are the most promising.
\end{abstract}

Keywords: systematic review; EMDR; somatoform disorders; MUS; adaptive information processing

$\mathbf{T}$ his review focuses on eye movement desensitization and reprocessing (EMDR) as a treatment for patients with chronic physical symptoms for which no treatable medical cause has been found or for whom regular treatment has not ameliorated the symptoms. This includes patients with somatoform disorders, functional syndromes, and physical symptoms after injury or illness from which the patient has (contrary to expectations) not recovered.

\section{Medically Unexplained Symptoms and Somatoform Disorders}

The magnitude of the problem of medically unexplained symptoms (MUS) is considerable given that about $25 \%-50 \%$ of all presentations to primary and secondary care are a consequence of somatic symptoms not well characterized by medical conditions (Kroenke \& Price, 1993). Patients who present with somatic symptoms for which there is no readily identified physical etiology pose a considerable diagnostic conundrum for physicians (McFarlane, Ellis, Barton, Browne, \& Van, 2008) and are often difficult to treat and costly for society (Barsky, Orav, \& Bates, 2005). The effect of MUS on the sufferers' quality of life is generally as great as the effect of comparable diseases with clear organic origin (Ferrari \& Kwan, 2001).

The diagnosis of MUS is one by exclusion, and there are no positive criteria to decide whether a pattern of bodily complaints is essentially medically unexplained or indicates structural or other specified pathology (Henningsen, Zipfel, \& Herzog, 2007). Almost all medical specialties have their own MUS or functional disorder: for rheumatologists, prominent muscle pain and tenderness is fibromyalgia; for gastroenterologists, abdominal pain with altered bowel habit is irritable bowel syndrome; and for infectious-disease specialists, chronic fatigue and myalgia is a postviral or chronic fatigue syndrome (Wessely, Nimnuan, \& Sharpe, 1999). Prevalence of some functional syndromes (e.g. irritable bowel syndrome) is as high as $15 \%$, but reliable prevalence rates depend on established diagnostic criteria, which exist for only a few functional syndromes. Small variations in the criteria can produce large variations in prevalence rates; for example, whereas the prevalence for chronic fatigue syndrome in the general population is estimated to be $0.2 \%$, the prevalence for chronic unexplained fatigue is 9\% (Henningsen et al., 2007). 
According to the Diagnostic and Statistical Manual of Mental Disorders (DSM-IV-TR), when MUS cause significant distress or disability they may be classified as a somatoform disorder (American Psychiatric Association, 2000). The category of somatoform disorders include: (a) somatization disorder, (b) undifferentiated somatoform disorder, (c) conversion disorder, (d) pain disorder, (e) hypochondriasis, (f) body dysmorphic disorder (BDD), and (g) somatoform disorder not otherwise specified (American Psychiatric Association, 2000). Somatoform disorders are a heterogeneous group of conditions, and their clinical presentations vary greatly (Table 1 ).

The DSM-IV (including the somatoform disorder section) provides a system of phenomenological classification. However, classification of somatoform disorders according to the DSM-IV is less precise than is necessary for clinical and research purposes because of the following: (a) the variable interpretation of the thresholds of severity required for these disorders, (b) the substantial error in physicians' rating of MUS, (c) the requirement that symptoms are not better explained by some other psychiatric disorder, such as depression or anxiety, and (d) the lack of stated direction about how to deal with the considerable overlap between somatoform disorders and functional medical syndromes (Strassnig, Stowell, First, \& Pincus, 2006). Another problem with the $D S M-I V$ is that the attribution of a psychiatric diagnosis to MUS suggests a purely psychogenic cause, whereas biological factors are known to play a role in the etiology of MUS and it is not yet clear whether psychological factors are also involved in the origin of MUS.

Alternative strategies for the DSM-V have been proposed to resolve many of these problems, to allow for a dimensional approach avoiding the dichotomization of patients into medical or psychiatric categories (McFarlane et al., 2008), and to stimulate research in this important but fragmented clinical area that still lacks consensus regarding diagnostic definitions (Strassnig et al., 2006).

\section{MUS and Comorbidity}

The relationship between MUS and anxiety and mood disorders is an ongoing topic of study. It is reported that anxiety and depression are more prevalent in patients with functional somatic syndromes than in healthy controls or in patients with a medical disorder of known etiology (Henningsen, Zimmermann, \& Sattel, 2003). However, many cases of MUS also occur in the absence of a specific anxiety or depressive disorder. Anxiety and depression are only moderately correlated with somatic symptoms, supporting the view that somatic symptoms cannot simply be seen as a bodily expression of anxiety or depression, or as their psychophysiological consequence (McFarlane et al., 2008).

Andreski, Chilcoat, \& Breslau (1998) have determined that of all the psychiatric disorders, posttraumatic stress disorder (PTSD) is the one that has the strongest relationship with somatization and, in particular, with medically unexplained pain. Many patient

TABLE 1. Somatoform Disorders and Medically Unexplained Symptoms (MUS)

\begin{tabular}{ll}
\hline Somatoform disorder & Description of MUS \\
\hline $\begin{array}{l}\text { Somatization disorder } \\
\text { Undifferentiated somatoform disorder }\end{array}$ & $\begin{array}{l}\text { Multiple different MUS } \\
\text { MUS or syndromes: chronic fatigue syndrome, irritable } \\
\text { bowel syndrome, noncardiac chest pain, etc. }\end{array}$ \\
Conversion disorder & $\begin{array}{l}\text { Medically unexplained neurological symptoms, myoclonic } \\
\text { movements, psychogenic nonepileptic seizures }\end{array}$ \\
Pain disorder & $\begin{array}{c}\text { Chronic pain syndromes: phantom limb pain, chronic } \\
\text { regional pain syndrome, postwhiplash syndrome, } \\
\text { repetitive strain injury, fibromyalgia, tension headache, } \\
\text { migraine, etc. }\end{array}$ \\
$\begin{array}{l}\text { Health anxiety } \\
\text { Body dysmorphic disorder }\end{array}$ & $\begin{array}{l}\text { Preoccupation with appearance } \\
\text { Not otherwise specified somatoform disorder }\end{array}$ \\
$\begin{array}{l}\text { MUS, health anxiety, and preoccupation with aspects of } \\
\text { appearance or other physical features (e.g., odor) not } \\
\text { fulfilling criteria of any of the other somatoform } \\
\text { disorders }\end{array}$
\end{tabular}


groups with MUS show high rates of PTSD. For example, clinically significant levels of PTSD symptoms were seen in $57 \%$ of a sample of fibromyalgia patients (Cohen et al., 2002). Marchetti et al. (2008) and Fiszman, Alves-Leon, Nunes, D’Andrea, \& Figueira (2004) found higher rates of actual PTSD (61.5\%) in adult patients with psychogenic nonepileptic seizures than in controls with epilepsy (27.3\%). Furthermore, PTSD is also associated with a number of functional syndromes - that is, undifferentiated somatoform disorders-including irritable bowel syndrome (Savas et al., 2008). In their review on the relationship between MUS and trauma, Roelofs and Spinhoven (2007) evaluated the evidence for this relationship, demonstrating that trauma in the form of childhood sexual, physical, and/ or emotional abuse is particularly common in patients with MUS, although it is not universally found. The association of MUS with PTSD and childhood trauma suggests that unresolved PTSD symptoms may contribute to the level of somatic symptoms, and this emphasizes the need to consider directed and primary treatment of PTSD in rehabilitation programs for pain/somatic symptoms (Roth, Geisser, \& Bates, 2008).

\section{Evidence-Based Treatment}

Although psychological factors are regarded as central to MUS, patients with these problems have typically been managed within a medical setting, and referrals to mental health services are relatively rare. Interest in the psychological treatment of MUS has increased since the late 1980s, leading to the development of psychological interventions for these conditions. Although different treatment strategies can be applied, there is general agreement that a cognitive behavioral therapy (CBT) approach is useful in understanding and treating patients with MUS. Systematic reviews of treatment studies have validated the efficacy of CBT in a wide range of MUS (Allen, Escobar, Lehrer, Gara, \& Woolfolk, 2002; Henningsen et al., 2007; Kirmayer, Groleau, Looper, \& Dao, 2004; Kroenke \& Swindle, 2000; Looper \& Kirmayer, 2002). The overall conclusion of the reviews is for a modest effect size of CBT for MUS - that is, smaller than for anxiety and depressive disorders, but nevertheless clinically significant.

For some of the functional somatic syndromes and somatoform disorders, selective serotonin reuptake inhibitors (SSRIs) have also proven effective. For example, hypochondriasis and BDD (the two somatoform disorders sharing features with obsessive compulsive disorder) can be successfully treated with SSRIs (Greeven et al., 2007; Williams, Hadjistavropoulos, \& Sharpe, 2006). SSRIs and tricyclic antidepressants have also been successfully used in the treatment of chronic pain. Although CBT and SSRIs can be effective, new treatments need to be developed for those patients who do not benefit from these approaches.

\section{Use of EMDR in the Treatment of MUS}

\section{Theoretical Underpinning}

EMDR is widely used and acknowledged as a treatment modality for PTSD but is still in its experimental phase for other disorders, such as MUS. The theoretical model informing the application of EMDR interventions is the adaptive information processing (AIP) model (Shapiro, 2001). The AIP model posits that the foundations of current pathology are the emotions and physical sensations inherent in the unprocessed traumatic event and their inappropriate storage in memory (Ray \& Zbik, 2001; Shapiro, 2001; Stickgold, 2002).

We think that unprocessed traumatic experiences might maintain physically unexplained complaints in at least two ways. First, if the physical complaint (e.g., pain) originated during a traumatic experience (e.g., a car accident) and this experience has not been adequately processed, then situations that share characteristics with the original traumatic event (e.g., the sound of a car) can trigger the traumatic memory including the physical sensations (e.g., pain) that were experienced at the time of the traumatic event. In this case the physical complaint is the same as the physical sensation that was originally experienced and can best be defined as physical re-experience (van der Kolk $\&$ Fisler, 1995).

Apart from the physical complaint itself (e.g., fatigue), the meaning the physical complaint has for the patient (e.g., "I am helpless") may also remind the patient of a traumatic experience (e.g., being raped). This can be the event with which the physical complaint began, but it can also be a traumatic event unrelated to and taking place (long) before the beginning of the complaint. In this latter case it is not the physical complaint itself but the meaning of the complaint that activates the traumatic memory. Here we might speak of a cognitive and emotional re-experience. Both the posttraumatic stress that is the result of the triggering of the traumatic memory and the way the patient copes with this stressful situation may maintain the physical complaint and hinder recovery (van Rood \& Visser, 2008). 
If this is true, successful processing of the dysfunctional stored memory will lead to a reduction in the intensity of the somatoform complaint (de Roos \& Veenstra, 2009). When this complaint is the result of the dysfunctional storage of a somatosensory memory as part of a traumatic memory, we anticipate that successful reprocessing will have an immediate positive effect on the intensity of the complaint. When the traumatic memories are the impetus for (or help maintain) the dysfunctional significance attached to the complaint, it is expected that successful reprocessing will lead to a decrease in stress and more effective coping, positively influencing the circumstances for a more gradual recovery of the complaints (Grant $\&$ Threlfo, 2002).

\section{Types of Targets and EMDR Protocols}

Concerning case conceptualization, de Roos and Veenstra (2009) distinguished three types of EMDR targets in the treatment of patients with chronic pain. Although they specified these targets for chronic pain only, the targets can be assumed to also hold for patients with other somatic symptoms. Therefore, in the following paragraphs we have substituted the term "somatic memory" for the term "pain memory." The three types of targets are:

- Traumatic memory: a classic trauma or traumarelated memory ("Big T" trauma), such as the memory of a serious accident, life-threatening act of violence, or physical or sexual abuse that is physically or cognitively and emotionally re-experienced in the physical complaint.

- Somatic symptom-related memory: a current emotionally disturbing memory that is related to traumatic experiences with the somatic symptom or the traumatic consequences of the somatic symptom. Examples include having a pain attack, being abandoned by friends and family, and losing one's job ("small t" trauma, i.e., life experiences).

- Current somatic symptom: the in-session experience of the somatic symptom, and not the memory of an experience .

The EMDR standard protocol is used for the reprocessing of both traumatic and somatic symptom-related memories (Shapiro, 2001). When somatic sensations (associations) in the desensitization phase emerge during the targeting of the initial traumatic event or somatic symptom-related event, attention can be directed at the residual pain sensations themselves in order to process any memory fragments (Schneider, Hofmann, Rost, \& Shapiro, 2007; 2008).

If no index event can be identified, or if the processing of the traumatic memory or somatic symptom-related memory did not result in a reduction of the intensity of the physical symptom, the current somatic symptom itself may be targeted (de Roos \& Veenstra, 2009; Schneider et al., 2007). In this case the EMDR pain protocol can be used (de Roos \& Veenstra, 2009; Grant, 2000). The pain protocol has the same structure and consists of the same elements (i.e., assessment phase, desensitization, installation, etc.) as the EMDR standard protocol and also concerns full reprocessing. The protocols of Grant (2000) and de Roos and Veenstra (2009) differ in the level of detailed prescription on how to intervene during the desensitization phase.

Although the pain protocols are used in clinical practice, their use is still experimental and their contribution to the decrease in symptoms needs to be established in randomized controlled trials (RCTs).

\section{EMDR-Related Protocols}

What is referred to as "EMDR" differs between different publications. While the acronym suggests the use of the full EMDR standard protocol it sometimes seems to concern only the use of bilateral stimulation (BLS), occasionally combined with other non-EMDR techniques and interventions. An example of an EMDR-related protocol in the field of MUS is the protocol used in the RCT of Marcus (2008) for acute migraine headaches; in that study diaphragmatic breathing, cranial compression, and eye movements (BLS) were combined to abort a migraine attack in progress. Another example of an EMDR-related protocol used for specific MUS is eye movement desensitization (EMD) for the relief of pain, fatigue, anxiety, and depression in fibromyalgia patients (Friedberg, 2004), and for the relief of chronic pain (Hassard, 1995). In this EMD protocol, the therapist instructs the patient to focus on the sensation or feeling of the most salient or intense symptom while the therapist performs alternating hand taps or other forms of BLS. Before and after these BLS sets, current levels of pain, stress, and fatigue are rated. BLS is used both in the EMDR and the EMD procedure. The difference between EMDR and EMD is that in EMDR memory associations are elicited in each set, and the attention of the patient is only returned to the target memory after each channel of association has been completed. The EMD procedure on the other hand is more like systematic desensitization in that the attention of the patient is brought back to the target memory after each set. This difference might influence the rate of reprocessing and the subsequent effect on 
symptom reduction (F. Shapiro, personal communication, 2009).

\section{Method}

\section{Selection of Studies}

A search was conducted for published reports of EMDR for patients with somatoform disorders and MUS. A priori the decision was made to search only for studies published in full and in peer-reviewed journals between 1989 (when Shapiro published the first article on EMDR) and 2009.

A three-step search strategy (as employed in the present study) is recommended for systematic reviews of psychological therapy. First, we searched for studies in computerized databases and in the Journal of EMDR Practice and Research. Then, we used the ancestry method to find more studies on EMDR for somatoform disorders and MUS in reviews and articles reporting on empirical studies; that is, the reference sections of articles were inspected for relevant studies that had not yet been detected. Finally, all authors of the selected articles were sent an e-mail asking them whether they had articles on this topic submitted for publication.

Five bibliographic databases were selected and their results compared: PubMed, PsycINFO, EMBASE, Web of Science, and The Cochrane Library. For all databases, two concepts were combined: EMDR and MUS/somatoform disorders. For both concepts, extensive word variants were selected. A copy of the keywords is available from the authors. Apart from the somatoform disorders, the search included the following specific functional syndromes and pain disorders: fibromyalgia, irritable bowel syndrome, chronic fatigue syndrome, burning mouth syndrome, tinnitus, sick building syndrome, multiple chemical sensitivity syndrome, temporomandibular dysfunction, premenstrual syndrome, Gulf War syndrome, enuresis, psychogenic nonepileptic seizures, functional dyspepsia, phantom limb pain, chronic pelvic pain syndrome, abdominal pain, headache, post whiplash syndrome, chronic low back (neck or shoulder) pain, repetitive strain injury, myofascial pain syndrome, toothache, noncardiac chest pain, and complex regional pain syndrome.

The combination of both concepts yielded a total of 170 references: 66 references from PubMed, 29 references from EMBASE (of which 15 were unique), 53 references from Web of Science (of which 32 were unique), 25 references from The Cochrane Library (of which 14 were unique), 64 references from PsycINFO (of which 38 were unique), and 13 references from
CINAHL (of which 5 were unique). The Journal of EMDR Practice and Research resulted in an additional nine articles, of which seven were unique (prior to 2008).

Inspection of the reference sections resulted in two additional articles (Grant, 2000; Wilensky, 2006).

All authors were sent an e-mail asking whether they had related articles submitted for publication. In total, $65 \%$ of the authors responded, resulting in two additional articles (de Roos et al., in press; Mazolla et al., 2009). The other $35 \%$ of e-mails were returned as undeliverable.

\section{Inclusion and Exclusion Criteria}

The criteria for inclusion were: (a) intervention studies (case studies, case series, uncontrolled clinical trials, controlled trials, RCTs, and meta-analytic studies), (b) use of the full-standard EMDR procedure (Shapiro 2001), (c) publication in or submission for publication in a peer- reviewed journal, and (d) the aim of the intervention was to reduce the intensity or frequency of a somatic symptom or somatoform disorder.

Excluded were articles using EMDR-related protocols (i.e., EMD or BLS only) and articles investigating the effect of EMDR on experimentally induced physical sensations or on physiological parameters (e.g., heart rate, galvanic skin response).

Both authors (independently from each other) decided on the suitability for inclusion in the present review. Their results were compared and, in case of discrepancy, were discussed until agreement was reached.

Of the 181 identified articles, 171 did not fulfill the inclusion criteria, being theoretical reviews, studies not published in (or submitted to) a peerreviewed journal, or studies unrelated to the topic of the review; mostly these were neurobiological studies investigating eye movements. After discussion, a further 6 studies were excluded (Friedberg, 2004; Hassard, 1995; Hekmat, Groth, Rogers, 1994; Kelley, Benbadis, \& Adams, 2005; Marcus, 2008; Protinsky, Sparks, \& Flemke, 2001). In the remaining 16 articles the results of 110 patients were described. The same patient was described twice in the studies of Grant (2000) and Grant and Threlfo (2002), and also in the studies of Schneider et al. $(2007,2008)$; another patient described by Silver, Rogers, and Russell (2008) did not have a somatoform complaint; and 5 of 8 patients described by Kelley and Benbadis (2007) did not receive EMDR treatment. This left 102 unique patients who received EMDR for a somatic symptom. 


\section{Results}

\section{Types of Studies}

The 16 studies (Table 2) consisted of one RCT (Ray \& Page, 2002), two uncontrolled clinical studies (de Roos et al., in press; Mazolla et al., 2009), seven case series (Brown, McGoldrick, \& Buchanan, 1997; Grant \& Threlfo, 2002; Gupta \& Gupta, 2002; Kelley \& Benbadis, 2007; McGoldrick, Begum, \& Brown, 2008; Schneider et al., 2008; Wilensky, 2006), and six single cases (Chemali \& Meadows, 2004; Grant, 2000; Royle, 2008; Russell, 2008a, 2008b; Silver et al., 2008).
The one RCT used a pre-post cross-over design comparing the effect of one single session of EMDR with one session of hypnosis on pain intensity (Ray $\&$ Page, 2002). Patients $(n=17)$ were randomly assigned to receive either hypnosis followed by EMDR, or EMDR followed by hypnosis. A nontreatment ("waiting list" or "care as usual") control group was not included.

\section{Designs}

All studies used a pretest-posttest design. Follow-up data were collected for all but one large study (Mazolla et al.,

TABLE 2. Characteristics of the Studies in the Present Review

\begin{tabular}{|c|c|c|c|c|c|c|c|}
\hline Author(s) & $\begin{array}{l}\text { Study } \\
\text { type }\end{array}$ & $n$ & $\begin{array}{l}\text { Somatic } \\
\text { symptom }\end{array}$ & $\begin{array}{l}\text { Duration of somatic } \\
\text { symptom }\end{array}$ & $\begin{array}{l}\text { No. of } \\
\text { sessions }\end{array}$ & Follow-up & Outcome \\
\hline Grant, 2000 & Case & 1 & $\begin{array}{l}\text { Chronic } \\
\text { pain }\end{array}$ & 2 years & 2 & - & $(++)$ \\
\hline Grant and Threlfo, 2002 & $\mathrm{CS}$ & 3 & $\begin{array}{l}\text { Chronic } \\
\text { pain }\end{array}$ & 5 years $(2-10$ years $)$ & 9 & 2 months & + \\
\hline Ray and Page, 2002 & RCT & 17 & $\begin{array}{c}\text { Chronic } \\
\text { pain }\end{array}$ & - & 1 & $<1$ month & + \\
\hline Mazolla et al., 2009 & UCS & 38 & $\begin{array}{l}\text { Chronic } \\
\text { pain }\end{array}$ & 12 years & 12 & - & + \\
\hline Wilensky, 2006 & CS & 5 & PLP & $\begin{array}{l}9 \text { months } \\
\text { (1 week-3 years) }\end{array}$ & 5.6 & - & ++ \\
\hline Russell, 2008a & Case & 1 & PLP & 4 months & 2 & $<1$ month & ++ \\
\hline Schneider et al., 2008 & CS & 5 & PLP & $\begin{array}{l}8 \text { years } \\
\qquad(3-16 \text { years })\end{array}$ & 7 & $>1$ year & ++ \\
\hline de Roos et al., in press & UCS & 10 & PLP & 13.6 years $(1-39$ years $)$ & 5.9 & $>1$ year & + \\
\hline Chemali and Meadows, 2004 & Case & 1 & PNES & 3 years & 72 & 3 months & $(++)$ \\
\hline Kelley and Benbadis, 2007 & CS & 3 & PNES & $\begin{array}{l}5 \text { years, } 2 \text { years, } \\
\text { "many years" }\end{array}$ & 4.7 & $>1$ year & $(++)$ \\
\hline Silver et al., 2008 & Case & 1 & $\begin{array}{c}\text { Myclonic } \\
\text { move- } \\
\text { ment }\end{array}$ & $35-40$ years & 4 & 6 months & $(++)$ \\
\hline Brown et al., 1997 & CS & 7 & BDD & 7 years ( $2-24$ years) & 2.1 & $>1$ year & $(++)$ \\
\hline McGoldrick et al., 2008 & CS & 4 & ORS & $\begin{array}{l}15 \text { years, } 8 \text { years, } \\
\text { "long", unreported }\end{array}$ & 2.3 & $\begin{array}{c}6 \text { months - } \\
10 \text { years }\end{array}$ & $(++)$ \\
\hline Gupta and Gupta, 2002 & CS & 4 & SRDD & $\begin{array}{l}\text { Unreported }(n=3) ; 25 \\
\quad \text { years }(n=1)\end{array}$ & 4.3 & 6-12 months & $(++)$ \\
\hline Royle, 2008 & Case & 1 & CFS & 5 years & 9 & $>1$ year & $(++)$ \\
\hline Russell, 2008b & Case & 1 & $\begin{array}{c}\text { Combat } \\
\text { MUS }\end{array}$ & 1 year & 5 & 6 months & ++ \\
\hline
\end{tabular}

Note. UCS = uncontrolled clinical study; CS = case series; BDD = body dysmorphic disorder; CFS = chronic fatigue syndrome; MUS = medically unexplained symptoms; ORS = olfactory reference syndrome; PLP = phantom limb pain, PNES = psychogenic nonepileptic seizures, PTSD = posttraumatic stress disorder; SRDD = stress-related dermatological disorders. Number of sessions and duration of somatic symptom; mean or absolute number. Outcome $-=$ no effect, $+=$ effect but not clinically significant, $++=$ clinically significant effect ( $>2.5$ points on an 11-point scale). Effect reported as a clinical observation is indicated between brackets; formal outcome measures are not. 
2009), a case series (Wilensky, 2006), and one case study (Grant, 2000). The period of follow-up measurements ranged from less than 1 month (Ray \& Page, 2002; Russell, 2008a), 1 to 6 months (Chemali \& Meadows, 2004; Grant \& Threlfo, 2002; Russell, 2008a; Silver et al., 2008), 6 to 36 months (Brown et al., 1997; de Roos et al., in press; Gupta \& Gupta, 2002; Kelley \& Benbadis, 2007; McGoldrick et al., 2008; Royle, 2008; Schneider et al., 2008), to up to 10 years (McGoldrick et al., 2008) after treatment (Table 2).

\section{Participants}

The gender of patients was presented in all studies, except for that of Schneider et al. (2008), who later (personal communication) informed us that four of their five patients were male. Of our total sample of 102 patients, 37 (36\%) were male. Brown et al. (1997) and Mazolla et al. (2009) did not report the age of their patients. The average age of the remaining 57 patients was 43 (range 22-73) years.

\section{Type and Duration of Somatic Symptoms}

The type of complaints studied was diverse and the mean duration of the complaints ranged from 1 week (Wilensky, 2006) to 48 years (McGoldrick et al., 2008) (Table 2). Eight studies involving 80 patients reported the use of EMDR in the treatment of pain. Four of these studies reported on the effect of EMDR on patients with different types of chronic pain (59 patients), including neuropathic pain (4 patients), headaches (4 patients), and fibromyalgia (4 patients) (Mazolla et al., 2009). Ray and Page (2002) did not report the duration of the pain of their patients. For the remaining 42 patients the mean pain duration was 11.3 years.

The other four pain studies evaluated the use of EMDR for patients with phantom limb pain (PLP) (21 patients). The mean duration of pain for these patients was 8.5 years, ranging from 1 week (Wilensky, 2006) to 39 years (de Roos et al., in press).

Medically unexplained neurological complaints were the focus in three studies involving five patients; four patients had psychogenic nonepileptic seizures (Chemali \& Meadows, 2004; Kelley $\&$ Benbadis, 2007) and one patient had myoclonic movement of the upper body (Silver et al., 2008). The mean duration of complaints was 12.5 years (range 18 months to 36.5 years).

Furthermore, two studies reported on the effect of EMDR in the treatment of patients preoccupied with an aspect of their physical qualities. Seven patients were preoccupied with an aspect of their appearance (BDD) (Brown et al., 1997), and four patients with their odor, that is, olfactory reference syndrome (ORS)
(McGoldrick et al., 2008). The mean duration of complaints was 7.6 (range 1.5-24) years for BDD patients and 22.8 (range 8-48) years for ORS patients. Patients with ORS were older (range 31-60 years) than were patients with BDD (range teens to 30 s).

The remaining studies described the effect of EMDR in patients with stress-sensitive dermatological diseases, chronic fatigue syndrome, and war-related MUS (Table 2).

\section{Comorbid Psychiatric Disorders}

Eleven patients were assessed with a structured clinical interview for the classification of DSM-IV axis I psychiatric disorders (de Roos et al., in press; Schneider, 2008). Two (18\%) of these patients had comorbid disorders; the remainder had only MUS or a chronic pain disorder.

Clinical judgment was used in 35 patients (Brown et al., 1997; Chemali \& Meadows, 2004; de Roos et al., in press; Grant, 2000; Gupta \& Gupta, 2002; Kelley \& Benbadis, 2007; Ray \& Page, 2002; Russell, 2008a, 2008b; Silver et al., 2008). Of these 35 patients, 15 (43\%) had comorbid disorders.

Of all 46 patients for whom data on comorbidity were available, $14(30 \%)$ had at least one comorbid disorder: 11 (24\%) had PTSD, 9 (20\%) fulfilled the criteria for a major depression, and 2 patients had mixed anxiety and depression. Two patients, one with a stress-related dermatological disorder and the other with BDD, fulfilled the criteria for social phobia. Two patients had more than two comorbid disorders; one patient with PLP fulfilled not only the criteria for a pain disorder but also for obsessive-compulsive disorder, alcohol dependence, and adjustment disorder, and one patient with psychogenic nonepileptic seizures fulfilled the criteria for PTSD, depression, and unspecified learning disabilities.

\section{Treatment}

Treatment Rationale. In most of the case reports included in this review, the rationale for the use of EMDR in the area of the specific somatoform complaint was not explicitly stated; however, after reading the articles it emerged that in most studies EMDR was used because of the role that stressful or traumatic events were assumed to play in the etiology and maintenance of MUS. Others used EMDR because specific MUS complaints were similar to those in PTSD but with manifest symptoms in a specific sensory modality (e.g., vivid imagery/odors) (Brown et al., 1997; McGoldrick et al., 2008).

Use of EMDR Protocols and Targets. All nonpain studies used the standard EMDR protocol to process 
trauma-related memories related to the somatoform disorder or physical complaint. In almost all of the cases there was such an event, although not all patients were aware of its significance. When there was no traumatic memory, a recent trigger (McGoldrick et al., 2008) or the actual pain was used as primary target.

In 68 of the 80 patients treated for pain, the pain protocol was used on its own (Ray $\&$ Page, 2002) or in addition to the standard protocol (de Roos et al., in press; Grant \& Threlfo, 2002; Mazolla et al., 2009). The authors of the remaining pain studies only used the standard protocol. They specified that they dealt with the pain during the desensitization phase as it emerged during the targeting of the initial traumatic event (Grant, 2000; Russell, 2008a; Schneider et al., 2008; Wilensky, 2006).

The order in which the targets were processed differed between studies. Mazolla et al. (2009) targeted the pain sensations and only targeted the pain-related traumatic events when the patient was not in pain during the session, whereas Grant and Threlfo (2002) and de Roos et al. (in press) first processed the traumatic memories and then dealt with the remaining pain.

Frequency and Duration of Treatment. The number of sessions ranged from 1 to 72 (Chemali \& Meadows, 2004) and treatment duration ranged from 1 week (Ray \& Page, 2002) to 18 months (Chemali \& Meadows, 2004) (Table 2). If we disregard the study of Chemali and Meadows with 72 sessions, the remaining 101 patients had a mean number of 6.8 sessions.

Single or Combined Treatment. All but two studies (Ray \& Page, 2002; Silver et al., 2008) reported whether or not their patients received additional treatment. Most pain patients also used medication, and the dose was kept stable during treatment. The patient in the case study of Chemali and Meadows (2004) was hospitalized during EMDR treatment for depression and later received dialectic behavioral therapy for borderline personality disorder. The patients in the study of Mazolla et al. (2009) received no other treatments during the study; however, they were offered some relaxation and visualization techniques to cope with the distress that might arise between sessions. In the study of Ray and Page (2002), besides EMDR, their patients were offered a "vipassana technique" that is a visualisation technique using the image of a healing light directed to the body to dissolve pain and distress.

\section{Results of the Studies}

\section{Dropouts}

Some patients declined the offered treatment before it started; others dropped out during treatment. Kelley and Benbadis (2007) were the only authors to report the number of patients (43\%) who dropped out of the study before the start of EMDR treatment.

The number of patients who dropped out during treatment was reported by the authors of the uncontrolled clinical trials and ranged from 10\% (de Roos et al., in press) to $32 \%$ (Mazolla et al., 2009). The authors of the one RCT did not report the number of dropouts; however, from their data it can be concluded that two patients (12\%) did not finish the study (Ray \& Page, 2002). Schneider et al. (2008) reported that two patients stopped therapy before treatment was completed due to "real-world exigencies," that is, one was discharged from the hospital where he was treated, and the insurance company of another refused further reimbursement. One patient in the case series of Wilensky (2006) stopped treatment when he was almost pain free; the patient was satisfied with the results even though he still had PTSD symptoms.

\section{Primary Outcome Measurements}

Of the 16 studies, 8 used some formal outcome measurement. The effect of EMDR on pain intensity was measured using a numeric rating scale (NRS; 0-10) (de Roos et al., in press; Grant \& Threlfo, 2002; Russell, 2008a; Schneider et al., 2008; Wilensky, 2006) or a visual analog scale (VAS; $10 \mathrm{~cm}$ ) (Mazolla et al., 2009; Ray \& Page, 2002). Ray and Page (2002) and de Roos et al. (in press) used pain diaries. The other authors used an outcome measure consisting of one observation of the pain intensity at the time of assessment. Patients were asked "How much pain do you have right now?" Russell (2008b) used a NRS not only for pain intensity but also for various phantom limb sensations such as itching and tingling.

Of the studies using a formal outcome measurement, three studies ( $n=58$ ) reported on the effect of EMDR on chronic pain (Table 3). After treatment the mean pain intensity in the chronic pain patients had decreased by 1.2-2 points on a 10-point scale. Grant and Threlfo (2002) presented follow-up data showing that the results were maintained.

All PLP studies used formal outcome measurements. Since we had the results of all individuals who received EMDR for PLP $(n=21)$ we could compute the overall decrease in pain intensity (Figure 1). After treatment the overall mean pain intensity had decreased by $4.7(S E=0.69)$ points on a scale, where $0=$ no pain and $10=$ worst possible pain. The results were maintained at follow-up $(4.5 ; S E=0.8)$, at which time 11 patients $(52 \%)$ were pain free (pain intensity $<1$ ). 
TABLE 3. Mean Scores of Chronic Pain and PLP at Pretreatment, Posttreatment, and Follow-Up

\begin{tabular}{|c|c|c|c|c|c|c|}
\hline Type of complaint & Author(s) & $n$ & Pretreatment & Posttreatment & Follow-up & Period of follow-up \\
\hline \multirow[t]{3}{*}{ Chronic pain } & Grant and Threlfo, 2002 & 3 & 6 & 4.1 & 3 & 2 months \\
\hline & Ray and Page, 2002 & 17 & 4.7 & 3.5 & - & - \\
\hline & Mazolla et al., 2009 & 38 & 8 & 6 & - & - \\
\hline \multirow[t]{4}{*}{ Phantom limb pain } & Wilensky, 2006 & 5 & 7.8 & 1.2 & - & - \\
\hline & Russell, 2008a & 1 & 8 & 1 & 0 & 3 weeks \\
\hline & Schneider et al., 2008 & 5 & 9.1 & 2.8 & 2.8 & 12-24 months \\
\hline & de Roos et al., in press & 10 & 5 & 2.8 & 2.5 & 3-32 months \\
\hline
\end{tabular}

Note. Pain was measured on a numerical rating scale, where $0=$ no pain and $10=$ worst possible pain.
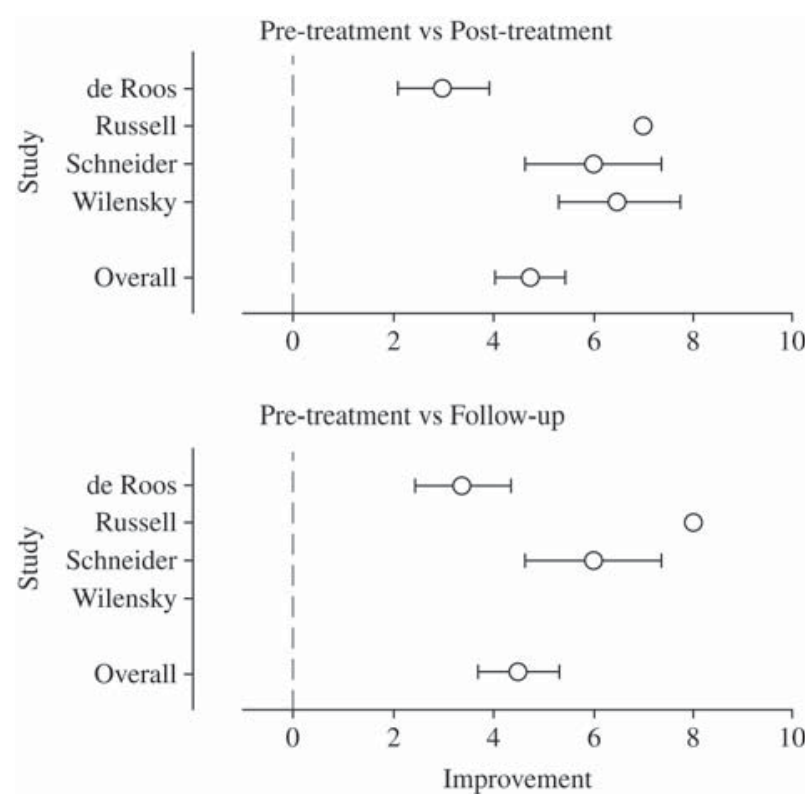

FIGURE 1. Changes in pain scores for phantom limb pain patients.

Besides the pain studies, the only other study using a formal outcome measurement was that of Russell (2008b). He reported on the effect of EMDR on warrelated MUS in a single case and used overall health status as measured on an 11-point NRS as outcome measure (higher scores indicating better health status). The overall health status of this patient improved from 1 to 6.5 posttreatment and to 8 at 6 -months follow-up.

The remaining eight studies did not use formal assessment to establish the effect of treatment but instead reported the clinically observed effect (Brown et al., 1997; Chemali \& Meadows, 2004; Grant, 2000; Gupta \& Gupta, 2002; Kelley \& Benbadis, 2007; McGoldrick et al., 2008; Royle, 2008; Silver et al., 2008) (Table 4).

\section{Secondary Outcome Measurements}

The secondary outcome measurements most often used were intensity of posttraumatic stress symptoms measured with the Impact of Event Scale (IES) and the intensity of depressive symptoms measured with the Beck Depression Inventory (BDI) (Table 5).

\section{The Impact of Event Scale (IES)}

Six studies used the IES to measure current subjective disturbance associated with a particular event. Different groups used different versions of the IES; the original IES (Horowitz, Wilmer, \& Alvarez, 1979), the IES-R, a revised version of the IES (Weiss $\&$ Marmer, 1997), and the Dutch IES 15-item version (van der Ploeg, Mooren, Kleber, Vander Velden $\&$ Brom, 2004) (Table 5). Scores on the IES and IES-R above 26 indicate moderate impact, and scores above 54 indicate severe impact associated with the specific event. The Dutch IES 15-item version uses different cut-off scores. However, the mean pretreatment IES scores of the patients assessed with the Dutch IES did not differ from normal controls. All IES scores decreased from pre- to posttreatment, were in the nonclinical range after treatment, and were maintained at follow-up (Table 5).

\section{The Beck Depression Inventory}

Six studies used the BDI to assess the intensity of depressive symptoms. The BDI consists of 21 items (range 0-63) (Beck, Ward, Mendelson, Mock \& Erbaugh, 1961). The BDI-II also consists of 21 items but has been modified according to the DSM-IV criteria (Beck, Steer \& Brown, 1996). The level of depression varied between studies. The patient described by Wilensky (2006) had severe depressive symptoms, and his score was in the severe range (30-63). The scores of the patients of Russell (2008a, 2008b) and the mean score of 
TABLE 4. Clinically Evaluated Effect of EMDR Treatment

\begin{tabular}{|c|c|c|c|c|}
\hline Author(s) & $n$ & Posttreatment & Follow-up & Period of follow-up \\
\hline Brown et al., 1997 & 7 & $\begin{array}{l}5 \text { patients free of BDD } \\
\text { symptoms, } 1 \text { partial } \\
\text { remission, } \\
1 \text { nonresponder }\end{array}$ & $\begin{array}{l}5 \text { patients free of BDD } \\
\text { symptoms, } 1 \text { partial } \\
\text { remission, } 1 \text { lost to } \\
\text { follow-up }\end{array}$ & $12-15$ months \\
\hline McGoldrick et al., 2008 & 4 & $\begin{array}{l}4 \text { patients complete } \\
\text { resolution } \\
\text { of all ORS symptoms }\end{array}$ & $\begin{array}{l}4 \text { patients complete } \\
\text { resolution of all ORS } \\
\text { symptoms }\end{array}$ & 6 months -10 years \\
\hline Chemali and Meadows, 2004 & 1 & $\begin{array}{l}\text { Seizure free (18 months } \\
\text { treatment, weekly sessions) }\end{array}$ & Seizure free & 3 months \\
\hline Kelley and Benbadis, 2007 & 3 & $\begin{array}{l}2 \text { patients free of PNES } \\
\text { symptoms, } 1 \text { nonresponder }\end{array}$ & $\begin{array}{l}2 \text { patients seizure free, } \\
1 \text { lost to follow-up }\end{array}$ & 18 months \\
\hline Silver et al., 2008 & 1 & Free of myoclonic movements & No incidents of shaking & 6 months \\
\hline Grant, 2000 & 1 & $\begin{array}{l}\text { Pain free, no more } \\
\text { sleeping problems }\end{array}$ & - & - \\
\hline Gupta and Gupta, 2002 & 4 & $\begin{array}{l}4 \text { patients free of } \\
\text { dermatological symptoms }\end{array}$ & $\begin{array}{l}4 \text { patients free of } \\
\text { dermatological symptoms }\end{array}$ & 6-12 months \\
\hline Royle, 2008 & 1 & $\begin{array}{l}\text { CFS; increase in energy levels } \\
\text { and decrease of hours sleep }\end{array}$ & $\begin{array}{l}\text { Normal level of activity } \\
\text { and working }\end{array}$ & 12 months \\
\hline
\end{tabular}

Note. $\mathrm{BDD}=$ body dysmorphic disorder; ORS = olfactory reference syndrome; PNES = psychogenic nonepileptic seizures; $\mathrm{CFS}=$ chronic fatigue syndrome .

the patients of Schneider et al. (2008) fell in the moderate to severe range (19-29) of depressive symptom severity, whereas the mean scores of the patients of de Roos et al. (in press) fell into the nonclinical range (0-9). In all studies, BDI scores decreased from pre- to posttreatment, were nonclinical or mild after treatment, and were maintained at follow-up (Table 5).

\section{Summary and Discussion}

This systematic review allows for the tentative conclusion that EMDR might play a role in the treatment of MUS. This conclusion is based on 16 studies: 13 cases or case series, 2 uncontrolled clinical trials, and 1 RCT. In total 102 patients received EMDR for a somatic complaint. The MUS studied fall in the following DSM-IV somatoform disorder categories: pain disorder, conversion disorder (unexplained neurological symptoms), BDD, and undifferentiated somatoform disorder. Of the undifferentiated somatoform disorders only chronic fatigue syndrome was studied, and this in one patient only.

All studies used a pretest-posttest design, and most studies provided follow-up data. With the exception of the 72 sessions in the study of Chemali and Meadows (2004), the duration of treatment was generally short enough to make it unlikely that other factors (e.g., changes in the patient and/or in life circumstances) influenced the outcome of these studies.
Because all but one of the studies were uncontrolled, it cannot be ruled out that the observed effect is a placebo effect or the result of spontaneous recovery. However, the latter explanation seems unlikely since most patients described in this review had physical complaints that had persisted for a long time and had often received various treatments without success.

Of all patients for whom data on comorbidity were available, only $24 \%$ fulfilled the criteria for PTSD according to the DSM-IV. This is less than expected based on ample reports that $50 \%$ or more of the patients with MUS have PTSD. The patients described in the present review also had less comorbid depressive disorders and anxiety disorders than could be expected. This raises the question whether these patients are in fact representative of the MUS and somatoform patients in general.

The treatment results in patients with MUS other than pain suggest that EMDR might be successfully used in the treatment of traumatized patients with BDD, ORS, and nonepileptic seizures. However, the many types of complaints and the small numbers of patients do not allow more specific conclusions.

On the other hand, the group of pain patients $(n=80)$ is large enough to discuss the results in more detail. The studies investigating the effect of EMDR in the mixed group of chronic pain patients $(n=58)$ showed less reduction in pain intensity than did the 
TABLE 5. Mean Scores on the Impact of Event Scale (IES) and Beck Depression Inventory (BDI) at Pretreatment, Posttreatment, and Follow-Up

\begin{tabular}{|c|c|c|c|c|c|c|c|c|c|}
\hline Author(s) & Symptom & $n$ & Pretreatment IES & Posttreatment IES & Follow-up IES & Pretreatment BDI & Posttreatment BDI & Follow-up BDI & Period of follow-up \\
\hline Wilensky, 2006 & PLP & 5 & $62(n=3)^{\mathrm{a}}$ & $22(n=3)$ & - & $34(n=1)$ & $11(n=1)$ & - & - \\
\hline Russell, 2008a & PLP & 1 & $39^{\mathrm{a}}$ & 13 & 8 & $19^{d}$ & 5 & 2 & 3 weeks \\
\hline Russell, 2008b & War-related MUS & 1 & $72^{\mathrm{a}}$ & 10 & 4 & $22^{\mathrm{e}}$ & 8 & 6 & 6 months \\
\hline $\begin{array}{l}\text { Schneider et al., } \\
\quad 2008\end{array}$ & PLP & 5 & $54^{\mathrm{b}}$ & 15 & 8 & $21^{\mathrm{e}}$ & 12 & 9 & $12-24$ months \\
\hline Silver et al., 2008 & Myclonic movement & 1 & $72^{\mathrm{b}}$ & 0 & - & $17^{\mathrm{e}}$ & 9 & & - \\
\hline $\begin{array}{l}\text { de Roos et al., } \\
\text { in press }\end{array}$ & PLP & 10 & $26^{c}$ & 18 & 21 & $8^{\mathrm{d}}$ & 3 & 6 & 3 months \\
\hline
\end{tabular}

Note. MUS = medically unexplained aymptoms; PLP = phantom limb pain.

aIES. bIES-R. ' IES-15 item version. ${ }^{\mathrm{d} B D I-I I . ~}{ }^{\mathrm{e} B D I}$ 
studies investigating the effect of EMDR in PLP $(n=21)$. Possible explanations for this might be the differences between these two patient groups. First, the mixed group of chronic pain patients is heterogeneous for the type of pain and the origin of the pain, whereas all the studies investigating the effect of EMDR on PLP included patients who had lost a limb (in most cases through a traumatic event). If EMDR is indeed more effective in patients with trauma-related MUS, one would expect better results in PLP patients than in a more varied group of pain patients. Second, the chronic pain and the PLP studies differed in the primary targets used. Two studies investigating the effect of EMDR on chronic pain used the actual pain sensations as primary target for EMDR (Mazolla et al., 2009; Ray \& Page, 2002), whereas all studies investigating the effect of EMDR on PLP first targeted the traumarelated memories. If the actual pain is used as target because of the lack of an index trauma, and if EMDR is more effective in trauma-related MUS, this might explain the less successful results obtained in the mixed chronic pain group. Finally, the one and only RCT investigating chronic pain patients showed a minor reduction in pain intensity; treatment in this latter study consisted of one session only. In the other studies exploring the effect of EMDR on pain, the mean number of sessions needed for a clinically relevant reduction in symptoms was 6.8 . This makes it likely that the duration of the study of Ray and Page (2002) was suboptimal and therefore does not allow us to draw conclusions about the efficacy of EMDR or hypnosis when this is offered as complete treatment.

From the preceding discussion we conclude that EMDR might have a positive and clinically relevant effect on pain intensity in PLP patients but that it is too early to draw even tentative conclusions about the usefulness of EMDR for chronic pain in general.

\section{Research Implications}

To assess the real effect of EMDR for PLP and other MUS, large studies of high validity (i.e., RCTs) are needed. However, before RCTs are designed, case studies might be used to establish whether a particular intervention can be associated with beneficial outcomes (McLeod, 2002). Some MUS that are associated with trauma (e.g., hypochondriasis and irritable bowel syndrome) have not yet been described in case studies. A beneficial effect of EMDR might be expected in these conditions if EMDR is indeed effective in the treatment of trauma-related MUS. When conducting a case study, certain methodological considerations can be taken into account to enhance its clinical and scientific relevance. Patients should be carefully selected, keeping in mind the question to be answered. When the potential use of a treatment is under study, then the selection of a typical case would be most informative. Furthermore, information should be collected from different sources, and the evidence should be presented separately from the investigator's interpretations of the evidence (McLeod, 2002). Data from case studies can be analyzed using statistical methods. Time-series analysis can be used to analyze data collected continuously across baseline and intervention phases (Borckardt et al., 2008); when there is only one pretest and one posttest measure it can still be determined whether the change in symptom severity is reliable and clinically significant (Jacobson \& Truax, 1991).

Case studies do not allow for generalization of the results to other groups of patients; RCTs are required for this. When designing an RCT for MUS certain points should be taken into consideration. Because the MUS patient group is extremely heterogeneous, one might consider recruiting patients not only on the basis of their physical symptom or somatoform disorder but also on some other specific patient characteristics, for example, patients with and without PTSD or traumatic experiences. In this way, the therapeutic utility of EMDR for specific patient subgroups can be more precisely defined.

Structured clinical interviews should be used to identify traumatic experiences and to classify symptoms according to the DSM-IV. Outcome measures should be reliable and valid. An outcome measure consisting of the mean intensity of the main somatic complaint as registered two to three times a day over a period of 1 or 2 weeks gives a more reliable estimation of the intensity of the complaint than the intensity of the complaint measured at only one single moment in time.

To interpret the effect of the EMDR intervention, it is necessary to specify the type of EMDR targets, the order in which they are focused on, and the specific protocols that are used. Every event from the somatic symptom's history that is still emotionally disturbing is, in principle, suitable as a target for EMDR. However, the question remains: which target should be selected first for optimal results? Examining the effects of different targeting approaches (i.e., traumatic memories versus the actual somatic symptom) on the rate and magnitude of somatic 
symptom reduction may help to address this question (Schneider et al., 2007).

In view of the significant changes that occur naturally over time it is important that appropriate control groups are included. Although the design of appropriate control and placebo conditions is a generic problem for psychological research, efforts should be made to use placebo techniques that generate similar expectations of improvement as the active treatment. A possible control condition for EMDR in the treatment of PLP might be relaxation training. If a placebo control is not feasible, a standardized control condition (e.g., care as usual) can be used.

Finally, because of the chronic course and episodic nature of some of the somatic complaints (e.g., irritable bowel syndrome), time between treatment and follow-up should be long enough to allow investigators to reasonably conclude whether the results are maintained.

\section{Clinical Implications}

This review shows that a considerable number of patients do not accept EMDR treatment for MUS or drop out during treatment. High dropout rates are not uncommon in studies investigating the effect of psychological interventions in MUS and seem highest when the rationale for treatment does not correspond with the ideas the patients have about the origin of their symptoms.

The high percentage of patients (43\%) who refused EMDR treatment in the study of Kelley and Benbadis (2007) might, for example, be explained by the fact that their patients sought help for their complaints in a medical setting (neurology clinic), suggesting that they expected to find a somatic solution for their somatic problem. In general, such patients tend to refuse psychological treatment when they suspect that this implies a psychological cause for their physical complaints. However, even patients who attribute their complaints to a somatic cause accept psychological treatment when it is clearly explained that such treatment is intended to reduce the negative consequences of their physical complaints that hinder recovery from their complaints (Speckens, van Hemert, Bolk, Hawton, \& Rooijmans, 1995).

However, even patients who are willing to consider a psychological explanation for their complaints need a credible treatment rationale to motivate them for treatment. The rationale for the use of EMDR in the area of the specific somatoform complaint remained unclear in most of the studies, which could be another reason for the high dropout rates found in some studies.

The tentative conclusion of the present review is that EMDR might be useful in the treatment of patients with MUS on the condition that the MUS is trauma related (i.e., the current complaint is etiologically linked to or maintained by unprocessed traumatic events or negative life experiences). The results of the studies described in this review show that processing these memories using the standard EMDR protocol can lead to a clinically relevant reduction of the physical and psychological symptoms.

It is not yet clear whether targeting the current physical sensations after successful processing of the traumatic memory results in further reduction of the physical complaint. However, the results of the studies of Mazolla et al. (2009) and Ray and Page (2002) suggest that the use of the current pain sensations as primary target in EMDR is not as effective as the use of a traumatic memory as primary target.

Of all 46 patients for whom data on comorbidity were available, $14(30 \%)$ had a comorbid disorder. After treatment with EMDR, not only did the intensity of the specific MUS decrease but the psychological symptoms also decreased; these results were maintained at follow-up. The implication of this finding is that it might be efficient to await the results of the EMDR treatment before starting treatments specific for comorbidity, such as CBT or SSRIs for depression or for anxiety disorders.

\section{Conclusion}

Until now the role of unprocessed traumatic memories in the etiology and maintenance of MUS has received insufficient attention, and most evidence-based treatments for MUS or somatoform disorders do not include interventions for the reprocessing of traumatic memories. This review shows that unresolved PTSD symptoms may play a role in the maintenance of physical symptoms and emphasizes the need to consider evidence-based treatment of PTSD (such as EMDR) in the treatment of somatic complaints. Given that somatoform disorders are relatively widespread, this finding has major implications for clinicians treating patients suffering from these conditions. However, because this fragmented clinical area still lacks agreedupon guidelines, it is important that clinicians and researchers cooperate from the beginning. In this way, the gap between clinical practice and research can be bridged, stimulating the exchange of knowledge 
and providing new insights. The many patients with MUS, their personal suffering, and the costs for society justify the funding of research investigating the applicability of EMDR as an evidenced-based treatment in MUS.

\section{References}

Allen, L. A., Escobar, J. I., Lehrer, P. M., Gara, M. A., \& Woolfolk, R. L. (2002). Psychosocial treatments for multiple unexplained physical symptoms: A review of the literature. Psychosomatic Medicine, 64, 939-950.

American Psychiatric Association. (2000). Diagnostic and statistical manual of mental disorders (4th ed., text revision). Washington, DC: Author.

Andreski, P., Chilcoat, H., \& Breslau, N. (1998). Post-traumatic stress disorder and somatization symptoms: A prospective study. Psychiatry Research, 79, 131-138.

Barsky, A. J., Orav, E. J., \& Bates, D. W. (2005). Somatization increases medical utilization and costs independent of psychiatric and medical comorbidity. Archives of General Psychiatry, 62, 903-910.

Beck, A. T., Steer, R. A., \& Brown, G. K. (1996). Manual for the Beck Depression Inventory (2nd ed.). San Antonio, TX: Psychological Corporation.

Beck, A. T., Ward, C. H., Mendelson, M., Mock, J., \& Erbaugh, J. (1961). An inventory for measuring depression. Archives of General Psychiatry, 4, 561-571.

Borckardt, J. J., Nash, M. R., Murphy, M. D., Moore, M., Shaw, D., \& O’Neil, P. (2008). Clinical practice as natural laboratory for psychotherapy research: A guide to case-based time-series analysis. American Psychologist, 63, 77-95.

Brown, K. W., McGoldrick, T., \& Buchanan, R. (1997). Body dysmorphic disorder: Seven cases treated with eye movement desensitization and reprocessing. Behavioural and Cognitive Psychotherapy, 25, 203-207.

Chemali, Z., \& Meadows, M. E. (2004). The use of eye movement desensitization and reprocessing in the treatment of psychogenic seizures. Epilepsy and Behavior, 5, 784-787.

Cohen, H., Neumann, L., Haiman, Y., Matar, M. A., Press, J., \& Buskila, D. (2002). Prevalence of posttraumatic stress disorder in fibromyalgia patients: Overlapping syndromes or post-traumatic fibromyalgia syndrome? Seminars in Arthritis and Rheumatism, 32, 38-50.

de Roos, C., Veenstra, A. C., de Jongh, A., den HollanderGijsman, M. E., van der Wee, N. J. A., \& van Rood, Y. R. (in press). Treatment of chronic phantom-limb-pain (PLP) using a trauma focused psychological approach: Ten cases. Pain research and management.

de Roos, C., \& Veenstra, S. (2009). EMDR pain protocol for current pain. In M. Luber (Ed.), EMDR scripted protocols, special populations, pp. 537-557. Springer Publishing Company: New York.
Ferrari, R., \& Kwan, O. (2001). The no-fault flavor of disability syndromes. Medical Hypotheses, 56, 77-84.

Fiszman, A., Alves-Leon, S. V., Nunes, R. G., D’Andrea, I., \& Figueira, I. (2004). Traumatic events and posttraumatic stress disorder in patients with psychogenic nonepileptic seizures: A critical review. Epilepsy and Behavior, 5, 818-825.

Friedberg, F. (2004). Eye movement desensitization in fibromyalgia: A pilot study. Complementary Therapies in Nursing and Midwifery, 10, 245-249.

Grant, M. (2000). EMDR: A new treatment for trauma and chronic pain. Complementary Therapies in Nursing and Midwifery, 6, 91-94.

Grant, M., \& Threlfo, C. (2002). EMDR in the treatment of chronic pain. Journal of Clinical Psychology, 58, 1505-1520.

Greeven, A., van Balkom, A. J., Visser, S., Merkelbach, J. W., van Rood, Y. R., van Dyck, R., et al. (2007). Cognitive behavior therapy and paroxetine in the treatment of hypochondriasis: A randomized controlled trial. American Journal of Psychiatry, 164, 91-99.

Gupta, M. A., \& Gupta, A. K. (2002). Use of eye movement desensitization and reprocessing (EMDR) in the treatment of dermatologic disorders. Journal of Cutaneous Medicine and Surgery: Incorporating Medical and Surgical Dermatology, 6, 415-421.

Hassard, A. (1995). Investigation of eye movement desensitization in pain clinic patients. Behavioural and Cognitive Psychotherapy, 23, 177-185.

Hekmat, H., Groth, S., \& Rogers, D. (1994). Pain ameliorating effect of eye movement desensitization. Journal of Behavior Therapy and Experimental Psychiatry, 25, 121-129.

Henningsen, P., Zimmermann, T., \& Sattel, H. (2003). Medically unexplained physical symptoms, anxiety, and depression: A meta-analytic review. Psychosomatic Medicine, 65, 528-533.

Henningsen, P., Zipfel, S., \& Herzog, W. (2007). Management of functional somatic syndromes. Lancet, 369, 946-955.

Horowitz, M. J., Wilmer, N., \& Alvarez, W. (1979). Impact of event scale: A measure of subjective stress. Psychosomatic Medicine, 41, 209-218.

Jacobson, N. S., \& Truax, P. (1991). Clinical significance: A statistical approach to defining meaningful change in psychotherapy research. Journal of Consulting and Clinical Psychology, 59, 12-19.

Kelley, S. D., \& Benbadis, S. (2007). Eye movement desensitization and reprocessing in the psychological treatment of trauma-based psychogenic non-epileptic seizures. Clinical Psychology and Psychotherapy, 14, 135-144.

Kelley, S. D., Benbadis, S. R., \& Adams, D. (2005). A community-based study of eye movement desensitization and reprocessing in the psychological treatment of trauma-based psychogenic seizures. Epilepsia, 46, 347.

Kirmayer, L. J., Groleau, D., Looper, K. J., \& Dao, M. D. (2004). Explaining medically unexplained symptoms. Canadian Journal of Psychiatry, 49, 663-672. 
Kroenke, K., \& Price, R. K. (1993). Symptoms in the community. Prevalence, classification, and psychiatric comorbidity. Archives of Internal Medicine, 153, 2474-2480.

Kroenke, K., \& Swindle, R. (2000). Cognitive-behavioral therapy for somatization and symptom syndromes: A critical review of controlled clinical trials. Psychotherapy and Psychosomatics, 69, 205-215.

Looper, K. J., \& Kirmayer, L. J. (2002). Behavioral medicine approaches to somatoform disorders. Journal of Consulting and Clinical Psychology, 70, 810-827.

Marchetti, R. L., Kurcgant, D., Neto, J. G., von Bismark, M. A., Marchetti, L. B., \& Fiore, L. A. (2008). Psychiatric diagnoses of patients with psychogenic non-epileptic seizures. Seizure, 17, 247-253.

Marcus, S. V. (2008). Phase 1 of integrated EMDR: An abortive treatment for migraine headaches. Journal of EMDR Practice and Research, 2, 15-25.

Mazolla, A., Calcagno, M. L., Goicochea, M. T., Pueyrredòn, H., Leston, J., \& Salvat, F. (2009). EMDR in the treatment of chronic pain. Journal of EMDR Practice and Research, 3, 66-79.

McFarlane, A. C., Ellis, N., Barton, C., Browne, D., \& Van, H. M. (2008). The conundrum of medically unexplained symptoms: Questions to consider. Psychosomatics, 49, 369-377.

McGoldrick, T., Begum, M., \& Brown, K. W. (2008). EMDR and olfactory reference syndrome: A case series. Journal of EMDR Practice and Research, 2, 63-68.

McLeod, J. (2002). Case studies and practitioner research: Building knowledge through systematic inquiry into individual cases. Counselling and Psychotherapy Research, 2, 265-268.

Protinsky, H., Sparks, J., \& Flemke, K. (2001). Eye movement desensitization and reprocessing: Innovative clinical applications. Journal of Contemporary Psychotherapy, 31, 125-135.

Ray, A. L., \& Zbik, A. (2001). Cognitive behavioral therapies and beyond. In C. D. Tollison, J. R. Satterhwaite, \& J. W. Tollsion (Eds.), Practical pain management (3rd ed., pp. 189-209). Philadelphia: Lippincott.

Ray, P., \& Page, A. C. (2002). A single session of hypnosis and eye movement desensitisation and reprocessing (EMDR) in the treatment of chronic pain. Australian Journal of Clinical and Experimental Hypnosis, 30, 170-178.

Roelofs, K., \& Spinhoven, P. (2007). Trauma and medically unexplained symptoms towards an integration of cognitive and neuro-biological accounts. Clinical Psychology Review, 27, 798-820.

Roth, R. S., Geisser, M. E., \& Bates, R. (2008). The relation of post-traumatic stress symptoms to depression and pain in patients with accident-related chronic pain. Journal of Pain, 9, 588-596.

Royle, L. (2008). EMDR as a therapeutic treatment for chronic fatigue syndrome (CFS). Journal of EMDR Practice and Research, 2, 226-232.
Russell, M. C. (2008a). Treating traumatic amputationrelated phantom limb pain: A case study utilizing eye movement desensitization and reprocessing within the armed services. Clinical Case Studies, 7, 136-153.

Russell, M. C. (2008b). War-related medically unexplained symptoms, prevalence, and treatment: Utilizing EMDR within the armed services. Journal of EMDR Practice and Research, 2, 212-225.

Savas, L. S., White, D. L., Wieman, M., Daci, K., Fitzgerald, S., Laday, S. S., et al. (2008). Irritable bowel syndrome and dyspepsia among women veterans: Prevalence and association with psychological distress. Alimentary Pharmacology and Therapeutics, 29, 115-125.

Schneider, J., Hofmann, A., Rost, C., \& Shapiro, F. (2007). EMDR and phantom limb pain: Theoretical implications, case study, and treatment guidelines. Journal of EMDR Practice and Research, 1, 31-45.

Schneider, J., Hofmann, A., Rost, C., \& Shapiro, F. (2008). EMDR in the treatment of chronic phantom limb pain. Pain Medicine, 9, 76-82.

Shapiro, F. (2001). Eye movement desensitization and reprocessing (EMDR): Basic principles, protocols, and procedures (2nd ed.). New York: Guilford Press.

Silver, S. M., Rogers, S., \& Russell, M. (2008). Eye movement desensitization and reprocessing (EMDR) in the treatment of war veterans. Journal of Clinical Psychology: In Session, 64, 947-957.

Speckens, A. E., van Hemert, A. M., Bolk, J. H., Hawton, K. E., \& Rooijmans, H. G. (1995). The acceptability of psychological treatment in patients with medically unexplained physical symptoms. Journal of Psychosomatic Research, 39, 855-863.

Stickgold, R. (2002). EMDR: A putative neurobiological mechanism of action. Journal of Clinical Psychology, 58, $61-75$.

Strassnig, M., Stowell, K. R., First, M. B., \& Pincus, H. A. (2006). General medical and psychiatric perspectives on somatoform disorders: Separated by an uncommon language. Current Opinion in Psychiatry, 19, 194-200.

van der Kolk, B. A., \& Fisler, R. (1995). Dissociation and the fragmentary nature of traumatic memories: Overview and exploratory study. Journal of Traumatic Stress, 8, 505-525.

van Rood, Y. R., \& Visser, S. (2008). Principes van cognitieve gedragstherapie bij patiënten met een somatoforme stoornis in de GGZ. Principles of cognitive behavior therapy in the treatment of patients with a somatoform disorder. In C. M. van der Feltz-Cornelis \& H. van der Horst (Eds.), Handboek somatisatie. Lichamelijk onverklaarde klachten in de eerste en tweede lijn [Handbook somatization: Medically unexplained symptoms in primary and secondary care] (2nd ed., pp. 269-291). Utrecht: Tijdstroom.

van der Ploeg, E., Mooren, T. T., Kleber, R. J., vander Velden, P. G., \& Brom, D. (2004). Construct validation of the Dutch version of the impact of event scale. Psychological Assessment, 16, 16-26. 
Weiss, D. S., \& Marmer, C. R. (1997). The impact of event scale-revised. In J. Wilson \& T. Keane (Eds.), Assessing psychological trauma and PTSD (pp. 412-428). New York: Guilford.

Wessely, S., Nimnuan, C., \& Sharpe, M. (1999). Functional somatic syndromes: One or many? Lancet, 354, 936-939.

Wilensky, M. (2006). Eye movement desensitization and reprocessing (EMDR) as a treatment for phantom limb pain. Journal of Brief Therapy, 5, 31-44.

Williams, J., Hadjistavropoulos, T., \& Sharpe, D. (2006). A meta-analysis of psychological and pharmacological treatments for body dysmorphic disorder. Behavior Research and Therapy, 44, 99-111.

Acknowledgments. The authors thank Jan W. Schoones (Walaeus Library, Leiden University Medical Centre) for his assistance with the literature search and valuable comments on the "Method" section.

Correspondence regarding this article should be directed to Yanda R. van Rood, Department of Psychiatry, Leiden University Medical Centre, The Netherlands. E-mail: yrvan rood@lumc.nl. 